\title{
Bridging the Gap in Personalized Oncology using Omics Data and Epidemiology
}

\author{
Dina El Harouni, Farah Nabil and Hassan Adwan* \\ Department of Pharmacology and Toxicology, German University in Cairo, Egypt
}

*Corresponding author: Hassan Adwan, Department of Pharmacology and Toxicology, Faculty of Pharmacy and Biotechnology, German University in Cairo GUC, Main Entrance Al Tagamoa Al Khames, New Cairo 11835, Egypt

Submission: 海 July 19, 2018; Published: 監 August 13, 2018

\begin{abstract}
As Personalized Medicine tailored the field of precision oncology, many challenges have been arising to fulfill the dream of a full personalized health integrated system in cancer therapy. Personalized oncology has been addressed through the past decades in multiple disease and various stages using high throughput technology. This review gives hand on recent advances of personalized oncology in several cancer disease models including leukemia, melanoma, breast cancer, lung cancer, colorectal cancer, and prostate cancer. Moreover, the review enumerates technology-based assessment of personalized biomarkers, including chip micro-array, organ on chip, and next generation sequencing. Meanwhile addressing challenges faced in implementing true personalized health cancer in oncology setting, this review focuses on bridging the gap between omics data analytics and epidemiology to overcome the true challenge of direct application.
\end{abstract}

Keywords: Personalized medicine; Precision oncology; Pharmacogenomics; Pharmacometabolomics; Pharmacomicrobiomics; Pharmacometrics; Pharmacokinetics; Clinical epidemiology

\section{Introduction}

Personalized medicine is the concept of tailoring a personalized treatment for each individual or group of individuals based primarily on their genomic composition as well as environmental and demographic factors [1]. While most researchers and scientists use the terms personalized medicine and precision medicine interchangeably, some argue that they are not the same. "Personalized" is the older term and "precision" is the newer and more accurate term to describe the concept according to the National Research Council. Personalized healthcare may appear to be a relatively new and revolutionary concept. However, personalization has been applied and acknowledged in the field of medicine long before the success of the Human Genome Project [2]. Personalized and/or precision medicine has gained widespread attention and awareness from the public soon after the completion of the Human Genome Project in 2003.

Since initial sequencing and human genome analysis was accomplished, a huge effort has been put into medical research focused on associating genomic variations with individual phenotypes [3]. There have been numerous breakthroughs in the field of precision oncology over the past few years for the most part due to genetic biomarkers, which are certain genes that may affect a person's response to therapy and their prognosis [4]. A true application was the women are now screening for the single nucleotide polymorphisms (SNPs) in the BRCA1 and/or BRCA2 gene which if found significantly increase the risk of breast cancer and ovarian cancer. The early detection of such biomarkers allows the women to administer preventive chemotherapy or consider a prophylactic operation [5].

Genetic polymorphisms and mutations in drug metabolizing enzymes, transporters, receptors, and other drug targets are linked to inter-individual differences in the efficacy and toxicity of medications as well as genetic disease risk factors. The highest impact on personalized medicine is often seen for drugs with a narrow therapeutic index, with important examples emerging from treatment with antidepressants, oral anticoagulants, and chemotherapeutics, which are metabolized by CYP2D6/CYP2C9, VKORC1, and TPMT, respectively. To apply the increasing amounts of pharmacogenomics knowledge to clinical practice, specific dosage recommendations based on genotypes will have to be developed to guide the clinician. Increases in efficacy and safety by the individualization of medical treatment may have benefits in financial terms, if information is presented to show that personalized medicine will be cost-effective in healthcare systems [6].

While the scientific community has largely accepted the utility of sequencing for research purposes, the use of the next-generation sequencing (NGS) technology in a clinical setting has yet to be fully addressed and applied clinically [7]. To effectively advance personalized medicine, it is necessary to be able to test for patients' genet- 
ic polymorphisms and/or mutations rapidly and conveniently. New technologies are evolving to transform diagnostic tests and translational research into routine practice. Laboratory methods as Chip microarray, NGS, and organ on chip analysis have been used as powerful tools in research within all collaborative field of science and omics analytics that would be covered extensively in this review.

\section{Advances in Personalized Medicine}

\section{Leukemia}

Remarkable improvements in survival rates have made childhood acute lymphoblastic leukemia (ALL) a success story within pediatric oncology. Despite the progress that has been observed relapses occur unpredictably, and treatment can be associated with acute and long-term toxicity. This has prompted efforts to better tailor ALL therapy in individual patients. Adaptation of personalized or precision medicine in childhood leukemia takes into consideration not only somatically acquired characteristics of tumor cells, but also inherent patient characteristics. Genome-wide association studies have identified inherited genetic polymorphisms that predispose to the development of leukemia, treatment response and outcome [8-11].

The identification of germline polymorphisms in thiopurine methyltransferase (TPMT), a gene encoding an enzyme responsible for the metabolism of thiopurines and impacting tolerance of 6-mercaptopurine (6-MP), is an example of an observation that has had important implications for optimizing chemotherapy dosing in individual patients. Those harbouring mutant TPMT alleles have reduced TPMT function and accumulate excessive active thiopurine metabolites. While these patients have been reported to have more favorable outcomes, they are at higher risk for developing myelosuppression and secondary malignancies and may be unable to tolerate full doses of 6-MP $[12,13]$. Some treatment groups have now recommended routine screening for TPMT activity, in order to tailor dosing of thiopurines accordingly [14].

Another aspect of personalizing therapy for children with ALL focuses on measures to prevent acute and long-term treatment-related toxicities. Hypersensitivity reactions are one of the most common side effects of asparaginase, which is a mainstay of ALL therapy. These reactions frequently lead to the production of neutralizing antibodies and have been associated with inferior outcomes [15]. Fernandez and colleagues have recently identified a risk for asparaginase hypersensitivity reactions and antiasparaginase antibodies in individuals with HLA-DRB1*07:01 alleles. This study demonstrates a strategy for a priori identification of patients predisposed to developing an allergic reaction to this important drug [16].

On another side glucocorticoid-induced osteonecrosis (ON) occurs in up to $20 \%$ of adolescent patients receiving ALL therapy and leads to significant morbidity [17]. While several well-defined clinical risk factors exist, such as age and corticosteroid exposure, recent efforts have also focused on identifying genomic predictors.
Chang et al. [18] recently reported alterations in genes in the glutamate receptor pathway as predictors for glucocorticoid associated ON in a large genome-wide association study that included 2285 children with ALL. Nevertheless, the individualized treatment of Glucocorticoids is still under research and extensive precision investigation.

In the meanwhile, Adult ALL have been under research for personalized therapy. More sophisticated diagnostic procedures, including immunophenotyping, cytogenetics, molecular genetics, and new genomics, have allowed the definition of new ALL sub-entities which, in some cases, has translated into specific therapies. A great achievement is the possibility of evaluating minimal residual disease (MRD), which can now be done in about 95\% of ALL patients. MRD is the most important prognostic factor and thus a major component of a personalized treatment algorithm $[19,20]$. Targeted therapy in Philadelphia chromosome-positive ALL (Ph+ALL) with tyrosine kinase inhibitors (TKI) and immunotherapy with monoclonal antibodies targeting surface antigens expressed on leukemic blast cells have extended the armamentarium [21]. Not Only ALL experience Personalized Medicine but also Acute Myeloid Leukemia (AML). Currently, treatment decisions for the majority of patients with AML depend on age and cytogenetic characteristics, and only occasionally on the use of a limited number of molecular alterations at diagnosis (mutations in FLT3, NPM1, and CEBPA, and possibly IDH1/2 mutations) [22].

\section{Melanoma}

The advances on melanoma molecular pathogenesis have opened a new insight for the management of advanced melanoma using personalized medicine. The development of novel therapies that target causative genetic events and improve disease free survival and overall survival was the key of application [23]. The selective BRAF kinase inhibitors (Vemurafenib and dabrafenib) are effective in BRAF mutant melanoma; MEK inhibitors (trametinib and cobimetinib) show efficacy against both BRAF- and KRAS/ NRAS-driven tumors; KIT inhibitors (imatinib, dasatinib, sunitinib and nilotinib) have demonstrated clinical responses in melanoma arising from acral, mucosal, and chronic sun-damaged cutaneous sites; and additionally, there are novel therapeutic monoclonal antibodies targeted against immunosuppressive molecules such as CTLA4, PD-1 and PD-L1 [24]. Therefore, molecular diagnostics are increasingly performed routinely in the diagnosis and management of patients with melanoma.

\section{Breast cancer}

Breast cancer is the most prevalent cancer in women. It is estimated that more than 500,000 annual deaths occur worldwide due to breast cancer [25]. The molecular subtyping of breast cancer is now possible due to the advancement in genomic technologies. The main molecular subtypes of breast cancer are: Estrogen receptor positive (ER+) which is further divided into the types Luminal A and Luminal B, Triple-negative which is human epidermal growth 
factor receptor 2 negative (HER2-), ER- and progesterone receptor negative (Pgr-), and HER2+. The triple negative and HER2+ are associated with bad prognoses while ER+ subtypes generally have good prognosis with Luminal A being the more favorable subtype [26]. HER+ is has seen the highest success with targeted therapy in the form of monoclonal antibodies: trastuzumab, pertuzum$\mathrm{ab}+$ trastuzumab; they are used as adjuvants in addition to chemotherapy [27].

Circulating tumor cells (CTCs) are a valuable biomarker in breast cancer patients. The monitoring and evaluation of the genomic changes in CTCs through whole genome sequencing (WGS) is helpful in the determination of the appropriate therapeutic intervention [28]. Another genomic biomarker of importance is the microRNA: a non-coding RNA that regulates gene expression. It was revealed that microRNA can trigger the release of chemotherapeutic agents from nanoparticles and into the cells [29]. Controlling the release time of the chemotherapeutic may reduce chemotherapy-induced side effects.

\section{Lung cancer}

Lung cancer is the leading cause of cancer-related death. It is estimated that lung cancer kills more than 1 million people every year. There are different subtypes of lung cancer. The first is NonSmall Cell Lung Carcinoma (NSCLC) which is further divided into the two subtypes Adenocarcinoma (ADC) and Squamous Cell Carcinoma (SCC). The second subtype is Small Cell Lung Carcinoma (SCLC). NSCLC cases constitute an estimate of $85 \%$ of lung cancer cases globally [30]. Thanks to DNA whole exome sequencing (WES), a full catalogue of somatically acquired mutations in lung cancer is available. However, targeted therapy still remains a challenge since lung tumors exhibit intra-tumor heterogeneity [31].

The majority of lung cancer cases, mainly in ADC, have EGFR mutations and EML4-ALK fusion gene. There is a large population of lung cancer patients of the NSCLC subtype that have the ALK fusion gene. It has been a target for ALK inhibitors such as crizotinib which has proven successful especially in ADC cases where it has a response rate equal to $57 \%-74 \%$ and it caused fewer adverse effects in comparison with traditional chemotherapy [32]. Crizotinib has had massive success in NSCLC patients with ROS1 rearrangements as well with a response rate to be close to $80 \%$ [33].

\section{Prostate cancer}

Prostate cancer (CaP), indolent or aggressive, is the most common malignancy in men worldwide. Indolent cases, which are localized and slow-progressing are more common than aggressive ones that tend to be resistant to radiotherapy (RT) and prone to metastasis [34]. One of the earliest diagnostic biomarkers is Prostate Specific Antigen (PSA) that present in higher amounts than usual in cases of malignancy. Mutated Androgen Receptor (AR) has a pronounced link with prostate cancer incidence. Traditional therapies include surgical or pharmacological androgen deprivation therapy (ADT). Moreover, inhibition of AR signalling by the targeting of the
CYP17 enzyme such as: enzalutamide, abiraterone, apalutamide has been effective [35]. AR mutations and gene amplification are also common in castration resistant prostate cancer (CRPC): an aggressive form in which cells develop ADT resistance

by hyper-activating androgen signalling [36].

\section{Colorectal cancer}

Advances in the treatment of colorectal cancer have led to an improvement in survival from 12 months with fluorouracil monotherapy to approximately 2 years. However, there are significant molecular differences between tumors which can affect both prognosis and response to treatment. Personalized medicine aims to tailor treatment according to the characteristics of the individual patient, specifically for early metastatic prognosis and biomarker precision application to personalized treatments. The first true use of personalized medicine in mCRC was the clinical testing of KRAS mutations (which occur in approximately $45-50 \%$ of patients with CRC) [37]. Subsequently the anti-EGFR treatment is given only to patients who are KRAS wild type. However, not all patients who are KRAS wild type respond to anti-EGFR therapy and therefore there has been substantial research into other potential predictive biomarkers for future precision application [38].

After KRAS mutations, BRAF V600E mutations currently have the strongest evidence to support their use as a predictive biomarker for EGFR-targeted mAb activity [39]. Most but not all of the available evidence links BRAF V600E mutations with resistance to EGFR-targeted mAb therapy [40], and therefore it is still under extensive research.

\section{Challenges in Personalized Medicine}

Genomics-based personalized medicine has gained public recognition for its multiple achievements during a short time period. However, many obstacles hinder its practical clinical implementation. Since the realization of the HGP, the cost of genomic analysis has significantly decreased as has the time required to sequence an entire genome. This development augmented the amount of available genomic, transcriptomic, proteomic and metabolomic information into what is known as "big data": a term applied to datasets whose size or type is beyond the ability of traditional relational databases to capture, manage, and process the data. The rapid emergence of big data in the field poses challenges for bioinformaticians. Furthermore, the use of medical big data requires the integration of various data classes, such as electronic health records (EHR), epidemiological studies, clinical trials, clinical registries, biobank data, multi-omics [41]. One merged, comprehensive, international network would bridge the gap between bioinformatics and clinical practice, facilitating the successful application of personalized healthcare in which effective communication between clinicians and bioinformaticians is crucial [42].

The article "Seven Questions for Personalized Medicine" discussed how PM is striving to fulfill "unrealistic" expectations set by the public. Authors argue that PM is not inclusive as the cost of tar- 
geted therapy is afforded only by the rich in developed countries; given that most current personalized therapies are cost-ineffective. Another argument states that the semi-complete dependence on genomics for the development of targeted therapies will not produce reliable predictions because the genome of an individual does not necessarily play a more important role in one's health than one's socioeconomic background and environment. Access to large omics (genomics, transcriptomics, proteomics, epigenomic, metagenomics, metabolomics, nutriomics, etc.) data has revolutionized biology and has led to the emergence of systems biology for a better understanding of biological mechanisms. Traditional observational epidemiology or biology alone are not sufficient to fully elucidate multifaceted heterogeneous disorders, and this directly limits all prevention and treatment pursuits for such diseases [43]. It is widely recognized that multiple dimensions must be considered simultaneously to gain understanding of biological systems [44]. Therefore, there is an urgent need to bridge the gap between advances in high-throughput technologies and the ability to manage, integrate, analyze, and interpret omics data to progress in personalized medicine [45].

As medicine begins to embrace genomic tools that enable more precise prediction and treatment disease, which include "whole genome" interrogation of sequence variation, transcription, proteins, and metabolites, the fundamentals of genomic and personalized medicine will require the development, standardization, and integration of several important tools into health systems and clinical workflows. These tools include health risk assessment, family health history, and clinical decision support for complex risk and predictive information. Together with genomic information, these tools will enable a paradigm shift to a comprehensive approach that will identify individual risks and guide clinical management and decision making, all of which form the basis for a more informed and effective approach to patient care. The ultimate challenge is the clinical application for improved patient care. In fact, many physicians are unprepared to incorporate personal genetic testing into their practice and it is unclear how to best apply research results to improve patient care [46]. Thus, bioinformatics can have the greatest clinical impact is in pharmacogenomics.

Bioinformatics lies as a bridge to implement parts of the clinical application in Personalized treatment. Bioinformatics also translates discoveries to the clinic by disseminating discoveries through curated, searchable databases like PharmGKB, dbGaP, PacDB and FDA AERS [47]. Finally, there are challenges and opportunities for bioinformatics to integrate with the electronic medical record (EMR). For example, the Bio Bank system at Vanderbilt links patient DNA with a deidentified EMRs to provide a rich research database for additional translational research in disease-gene and druggene associations [48]. Ultimately, bioinformatics needs to develop methods that integrate the genome in the clinic and allow physicians to use personalized medicine in their daily practice.

\section{Clinical Integration in Personalized Medicine}

The advance of precision/personalized medicine heavily re- lies on the ability to study biological phenomena at omics levels although the practice of precision/personalized medicine does not use only omics data and knowledge. This is because molecular characteristics obtained from omics data can classify diseases and identify subpopulation of patients suitable to certain common treatment more precisely. Following this trend, many of the emerging fields of large-scale data-rich biology are designated by adding the suffix '-omics' onto previously used terms. Specifically, pharmacogenomics, metabolomics, proteomics and others contribute to the glowing era of personalized treatment.

\section{Pharmacogenomics}

Pharmacogenomics is the study of how a person's response to drugs is affected by his/her genetic makeup. It combines pharmacology (the science of drugs) and genomics (the study of genes and their functions) to develop effective, safe medications and doses that will be tailored to a person's genetic makeup. The rapid accumulation of knowledge on genome-disease and genome-drug interactions has also impelled the transformation of pharmacogenetics into a new entity of human genetics, namely, pharmacogenomics. Enabled by high-throughput technologies in DNA analysis, genomics introduces a further dimension to individualized predictive medicine. Determining an individual's unique genetic profile in respect to disease risk and drug response will have a profound impact on understanding the pathogenesis of disease, and it may enable truly personalized therapy. This concept of therapy with the right drug at the right dose in the right patient has emerged as an urgent requirement among several studies on adverse drug effects in hospitalized patients $[49,50]$.

Technological progress in analyzing millions of genes and intergenic variants in the form of single nucleotide polymorphisms (SNP) and copy number variants per individual has accelerated our comprehension of individual differences in genetic makeup. Genome-wide association studies (GWAS) have successfully identified common genetic variations associated with numerous complex diseases.

\section{Pharmacometabolomics}

Metabolomics is defined as "the systematic identification and quantification of the small molecule metabolic products (the metabolome) of a biological system (cell, tissue, organ, biological fluid, or organism) at a specific point in time." The discovery and application NMR-Spectroscopy in 1940s revolutionized metabolomics making the detection of metabolites in biological fluids a reality [43]. In 2003 the first metabolic web-based database METLIN was created by the Scripps Research Institute in the USA. It now contains millions of small molecules including exogenous drugs and metabolites. In 2007 the Human Metabolome Database (HMDB) was launched. It is an online and freely available comprehensive database of metabolites found in the human body.

Pharmacometabolomics studies the relationship between pharmaceutical agents and the metabolome [44]. Pharmacometabolomics aims to create a metabolic fingerprint for each patient to determine therapeutic outcomes in terms of efficacy, adverse drug 
reactions and dosing. Metabolic profiles do not only differ according to a person's genome but also according to their environment, population group, lifestyle and microbiome [45]. Metabolomic studies are necessary for the correct implementation of personalized oncology. It was found that cancer cells have different metabolic requirements than normal cells [46]. Tumors alter their metabolism as a defense mechanism. This was shown in cancer settings including glycolysis [47], one carbon metabolism [48], and a multimetabolome project in Breast cancer therapy [49].

\section{Pharmacoproteomics}

Pharmacoproteomics, essentially a sub-discipline of functional pharmacogenomics, is a study of how the protein content of a cell or tissue changes qualitatively and quantitatively in response to treatment or disease, what the protein and protein-ligand interactions are in related to drug response, and how a person's protein variants in quality and quantity affect a person's response to a drug Protein roles are diverse, and mass spectrometry-based proteomics has established sophisticated tools and instruments that can identify proteins and measure the changes in protein levels, posttranslational modifications (e.g., kinase signaling), localization, and protein-protein or drug-protein interactions.

A leading study in the pharmacoproteomic field, described by Ong et al. [50], utilized quantitative proteomic analysis of SILAC-labeled cell lysates to identify specific protein interactions and targets of small molecules, including kinase inhibitors and immunophilin binders. Furthermore, identification of multiple protein targets may lead to novel combinatorial therapies, particularly in cancer, similar studies are emerging in the field [51] such as activated B cell-like diffuse large B cell lymphoma (ABC DLBCL, and more are yet to come.

\section{Pharmacomicrobiomics}

Microbiomics is the study of the human microbiome, the community of microbes that colonize the body. The human microbiome is influenced by a multitude of factors such as: age, gender, diet, socio-economic status, environment, health status, genotype and drug intake. Pharmacomicrobiomics is the interdisciplinary field that merges pharmacology, microbiology, and genomics. It studies the effect a host's microbiome has on xenobiotics' metabolism. In 2008 the Human Microbiome Project was launched by the National Institute of Health (NIH) in the USA. The project involved a comprehensive analysis of the human microbiome and how it affects one's health and susceptibility to diseases. Analysis of the microbiome is usually performed using next generation sequencing (NGS) techniques such as: shotgun metagenomic sequencing (SMS), 16S RNA sequencing, microbial whole genome sequencing (MWGS), and microbial metatranscriptomics.

A recent study showed the role of the microbiome on the response of metastatic melanoma patients to immunotherapy targeting programmed cell death protein 1 (PD-1)/ programmed cell death 1 ligand 1 (PD-L1). The results showed that responders had higher levels of bacterial genus Faecalibacterium while patients who did not respond well treatment had bacteria of the order Bacteroidales in their fecal samples [51]. These findings can be utilized as a basis for patient stratification when it comes to candidacy for immunotherapy [52]. Another study showed the chemotherapeutic agent gemcitabine seemed to experience high resistance by cancer cells when elevated levels of Escherichia coli were detected as the volume of tumors increased and the OS was decreased [53].

\section{Pharmacometrics}

Pharmacometrics is the application of mathematical and statistical methods to pharmacotherapy with the aim of describing or predicting concentrations, associated physiologic effects and clinical impacts. Pharmacometrics has become progressively a key science in the drug development process by implying the development of pharmacokinetic (PK) or PK/PD or PK/PD/PG models (PD, pharmacodynamics; $\mathrm{PG}$, pharmacogenomics) that provide knowledge about the behavior of a drug and how it can be optimally used. Additionally, when applied pragmatically, PM can also be an efficient, powerful and informative science in clinical settings, when adverse events or hazardous efficacy render individual dose adjustments necessary. In this chapter, the readers will find practical information about different approaches (population pharmacokinetics, Bayesian data analysis, etc.) usually used in PM, and examples of effective applications in clinical routine activity [54]. Integration of the modelling basis of Pharmacometrics could serve along with big data a major shift in precision medicine toward direct application.

\section{Epidemiology in Personalized Medicine}

Epidemiology is "the study of the distribution and determinants of health-related states or events in specified populations, and the application of this study to the control of health problems" according to the Centre for Disease Control. Nowadays, medicine is moving towards an evidence-based approach made possible by epidemiological studies which facilitate the identification of trends, patterns and emerging public health issues, and help us evaluate the outcome of different interventions [55].

One of the main focuses of epidemiology is how does one's socioeconomic status (SES) affects their health. It was found that high SES is linked to better health in both curable and incurable diseases. In multiple cancers, low SES is associated with late stage diagnosis and resultant poor prognosis. Another important epidemiological interest is race/ethnicity as a social determinant of health. For instance, studies reveal that minority racial groups in the USA are more likely to be diagnosed with late stage cancers and less likely to receive/continue treatment which significantly decreased their OS. This suggests that the key to reducing health disparities is population-based interventions. These kinds of interventions may seem to oppose the idea of personalized healthcare. However, PM strives to stratify patients into populations based on their environment, SES, lifestyle, and phenotype. Epidemiological studies are a fundamental basis for population-based interventions since they supply the 
data needed for patient classification into subpopulations. While genomics has revolutionized PM, they alone cannot be the answer. Full reliance on genomic studies arguably increases health disparities and perpetuate misconceptions about race [56].

Population based interventions are the answer to bridging the gap between the rich and poor and promoting health equity between races and ethnicities. They have also proven their success in disease prevention. And it could not have happened without the data obtained through extensive epidemiological studies. With that said, we come to the conclusion that epidemiology is essential for implementation of curative and preventive personalized medicine [42].

There are increasing number of huge cohort studies and cohort consortiums with long term follow-up. Many cohorts provide unique opportunities to address the effect of various demographic, lifestyle, genomic, molecular, clinical, as well as psychosocial factors on cancer outcomes. For example, the Prostate, Lung, Colorectal and Ovarian (PLCO) Cancer Screening Trial is a large population based randomized trial with extensive follow-up. By collecting biologic materials and risk factor information from trial participants before the diagnosis of disease, an ongoing PLCO component, the Etiology and Early Marker Studies (EEMS) is being added. Efforts can be undertaken to link the epidemiologic data with electronic medical and health records to further address the patient's outcomes.

Pharmaco-genetic-epidemiology studies can be nested on these life span cohorts. Recent advances in genomic research have demonstrated a substantial role for genomic factors in predicting response to cancer therapies. Translational cancer research is interdisciplinary and trans-disciplinary by nature. Numerous suggestions and recommendations have been making for multidisciplinary collaborations and partnerships to identify and fill the knowledge gaps. Much less attention has been paid to how to prepare the scientists for trans-disciplinary research. In fact, multidisciplinary training is a prerequisite for the next generation researchers who want to be fully capable to conduct translational cancer research. The next generation epidemiologists (NGEs) may have to obtain comprehensive knowledge of cancer epidemiology, molecular/genetic biology, statistics, and oncology or pathology [56]. Thus, NGE multi-disciplinary expertise is required. Nonetheless, knowledge integration is the key to clinical application of personalized medicine.

\section{Conclusion}

Precision medicine, characterized by genomic breakthroughs, has had a great impact on public health particularly in the last decade. Even though genomics has been the cornerstone of traditional personalized medicine and have made diagnosis, targeted therapy and prognosis inarguably easier through genomic biomarkers; other equally important field of scientific research such as: transcriptomics, proteomics, metabolomics, microbiomics, social and molecular epidemiology, pharmacometrics and bioinformatics must be integrated into precision medicine research.
In order to facilitate the existence of an integrated PM network, an important gap must be addressed in clinical settings. Healthcare workers must be familiarized with the areas of bioinformatics and multi-omics. Another important gap that must be bridged is the disparity in availability of PM to high socio-economic groups relative to low socio-economic groups. Therefore, genomic tests must be made more accessible to be inclusive of people of all backgrounds. We also believe that investments in population-based interventions such as prevention screening for stratified populations in of utmost importance as we are moving towards a more prevention-focused era of personalized medicine.

\section{References}

1. Bates S (2010) Progress towards personalized medicine. Drug Discov 15(3-4): 115-120.

2. Moeini R, Memariani Z, Pasalar P, Gorji N (2017) Historical root of precision medicine: an ancient concept concordant with the modern pharmacotherapy. Daru 25(1): 7

3. Consortium IHGS, Lander ES, Linton LM, Birren B, Nusbaum C, et al (2001) Initial sequencing and analysis of the human genome. Nature 409(6822): 860-921.

4. Kalia M (2015) Biomarkers for personalized oncology: recent advances and future challenges. Metabolism 64(3) Suppl 1: S16-S21.

5. Pan Z, Xie X (2017) BRCA mutations in the manifestation and treatment of ovarian cancer. Oncotarget 8(57): 97657-97670.

6. Relling MV, Evans WE (2015) Pharmacogenomics in the clinic. Nature 526(7573): 343-350

7. 1000 Genomes Project Consortium, Abecasis GR, Altshuler D, Auton A Brooks LD, et al.(2010) A map of human genome variation from population-scale sequencing. Nature 467 (7319): 1061-1073.

8. Treviño LR, Yang W, French D, Hunger SP, Carroll WL, et al. (2009) Germline genomic variants associated with childhood acute lymphoblastic leukemia. Nat Genet 41(9): 1001-1005.

9. Papaemmanuil E, Hosking FJ, Vijayakrishnan J, Price A, Olver B, et al. (2009) Loci on 7p12.2,10q21.2 and 14q11.2 are associated with risk of childhood acute lymphoblastic leukemia. Nat Genet 41(9): 1006-1010.

10. Prasad RB, Hosking FJ, Vijayakrishnan J, Papaemmanuil E, Koehler R, et al. (2010) Verification of the susceptibility loci on 7p12.2,10q21.2, and 14 q11.2 in precursor B-cell acute lymphoblastic leukemia of childhood. Blood 115(9): 1765-1767.

11. Sherborne AL, Hosking FJ, Prasad RB, Kumar R, Koehler R, et al. (2010) Variation in CDKN2A at 9p21.3 influences childhood acute lymphoblastic leukemia risk. Nat Genet 42(6): 492-494.

12. Schmiegelow K, Forestier E, Kristinsson J, Söderhäll S, Vettenranta K, et al. (2009) Thiopurine methyltransferase activity is related to the risk of relapse of childhood acute lymphoblastic leukemia: results from the NOPHO ALL-92 study. Leukemia 23(3): 557-564.

13. Relling MV, Hancock ML, Boyett JM, Pui CH, Evans WE (1999) Prognostic importance of 6-mercaptopurine dose intensity in acute lymphoblastic leukemia. Blood 93(9): 2817-2823.

14. Relling MV, Dervieux T (2001) Pharmacogenetics and cancer therapy. Nat Rev Cancer 1(2): 99-108.

15. Pui CH, Sandlund JT, Pei D, Campana D, Rivera GK, et al. (2004) Improved outcome for children with acute lymphoblastic leukemia: results of total therapy study XIIIB at St Jude children's research hospital. Blood104(9): 2690-2696. 
16. Fernandez Vina MA, Hollenbach JA, Lyke KE, Sztein MB, Maiers M, et al. (2012) Tracking human migrations by the analysis of the distribution of HLA alleles, lineages and haplotypes in closed and open populations. Philos Trans R Soc Lond B Biol Sci367(1590): 820-829.

17. Veerman AJ, Kamps WA, Van den Berg H, van den Berg E, Bökkerink JP, et al. (2009) Dexamethasone-based therapy for childhood acute lymphoblastic leukaemia: results of the prospective Dutch Childhood Oncology Group (DCOG) protocol ALL-9 (1997-2004). Lancet Oncol 10(10): $957-$ 966.

18. Xue Y, Lameijer EW, Ye K, Zhang K, Chang S, et al. (2016) Precision medicine: What challenges are we facing? Genomics Proteomics Bioinformatics 14(5): 253-261.

19. Brüggemann M, Schrauder A, Raff T, Pfeifer H, Dworzak M, et al (2010) Standardized MRD quantification in European ALL trials: proceedings of the Second International Symposium on MRD assessment in Kiel, Germany, 18-20 September 2008. Leukemia 24(3): 521-535.

20. Campana D (2010) Minimal residual disease in acute lymphoblastic leukemia. Hematol Am Soc Hematol Educ Progr 2010: 7-12.

21. Dombret H, Gabert J, Boiron JM, Rigal Huguet F, Blaise D, et al. (2002) Outcome of treatment in adults with Philadelphia chromosome-positive acute lymphoblastic leukemia-results of the prospective multicenter LALA-94 trial. Blood 100(7): 2357-2366.

22. Papaemmanuil E, Moritz G, Bullinger L, Verena IG, Paschka P, et al (2016) Genomic classification and prognosis in acute myeloid leukemia. N Engl J Med 374(23): 2209-2221.

23. Niezgoda A, Niezgoda P, Czajkowski R (2015) Novel approaches to treatment of advanced melanoma: a review on targeted therapy and immunotherapy. Biomed Res Int 2015: 851387.

24. Robert C, Long GV, Brady B, Dutriaux C, Maio M, et al. (2015) Nivolumab in previously untreated melanoma without BRAF mutation. N Engl J Med 372(4): 320-330

25. Tao Z, Shi A, Lu C, Song T, Zhang Z, et al. (2015) Breast cancer: epidemiology and etiology. Cell Biochem Biophys 72(2): 333-338.

26. Bettaieb A, Paul C, Plenchette S, Shan J, Chouchane L, et al. (2017) Precision medicine in breast cancer: reality or utopia? J Transl Med 15(1): 139.

27. Pinto AC, Ades F, De Azambuja E, Piccart Gebhart M (2013) Trastuzumab for patients with HER2 positive breast cancer: delivery, duration and combination therapies. Breast 22(Suppl 2): S152-S155.

28. Gulbahce N, Magbanua MJM, Chin R, Agarwal MR, Luo X, et al. (2017) Quantitative whole genome sequencing of circulating tumor cells enables personalized combination therapy of metastatic cancer. Cancer Res 77(16): 4530-4541.

29. Chan CWH, Law BMH, So WKW, Chow KM, Waye MMY (2017) Nove strategies on personalized medicine for breast cancer treatment: An update. Int J Mol Sci 18(11): E2423.

30. Molina JR, Yang P, Cassivi SD, Schild SE, Adjei AA (2008) Non-small cell lung cancer: epidemiology, risk factors, treatment, and survivorship. Mayo Clin Proc 83(5): 584-594.

31. Zhang Y, Wang DC, Shi L, Zhu B, Min Z, et al. ( 2017) Genome analyses identify the genetic modification of lung cancer subtypes. Semin Cancer Biol 42: 20-30.

32. Pao W, Girard N (2011) New driver mutations in non-small-cell lung cancer. Lancet Oncol 12(2): 175-180.

33. Mazières J, Zalcman G, Crinò L, Biondani P, Barlesi F, et al. (2015) Crizotinib therapy for advanced lung adenocarcinoma and a ROS1 rearrangement: results from the EUROS1 cohort. J Clin Oncol 33(9): 992-999.
34. Saini S (2016) PSA and beyond: alternative prostate cancer biomarkers. Cell Oncol (Dordr) 39(2):97-106.

35. Barbieri CE, Chinnaiyan AM, Lerner SP, Swanton C, MA Rubin (2017) The Emergence of precision urologic oncology: A collaborative review on biomarker-driven therapeutics. Eur Urol 71(2): 237-246.

36. Epstein JI, Amin MB, Beltran H, Lotan TL, Mosquera JM, et al. (2014) Proposed morphologic classification of prostate cancer with neuroendocrine differentiation. Am J Surg Pathol 38(6): 756-767.

37. Yamauchi M, Morikawa T, Kuchiba A, Imamura Y, Qian ZR, et al. (2012) Assessment of colorectal cancer molecular features along bowel subsites challenges the conception of distinct dichotomy of proximal versus distal colorectum. Gut 61(6): 847-854.

38. Zhao B,Wang L, Qiu H, Zhang M, Sun L, et al. (2017) Mechanisms of resistance to anti-EGFR therapy in colorectal cancer. Oncotarget8(3):3980-4000.

39. Pritchard CC, Grady WM (2011) Colorectal cancer molecular biology moves into clinical practice. Gut60(1): 116-129.

40. Mao C, Liao RY,Qiu LX, Wang XW, H Ding, et al.(2011) BRAF V600E mutation and resistance to anti-EGFR monoclonal antibodies in patients with metastatic colorectal cancer: a meta-analysis. Mol Biol Rep 38(4): 2219-2223.

41. Lee CH, Yoon HJ (2017) Medical big data: promise and challenges. Kidney Res Clin Pract 36(1): 3-11.

42. Khoury MJ, Gwinn ML, Glasgow RE, Kramer BS (2012) A population approach to precision medicine. Am J Prev Med 42(6): 639-645.

43. Loos RJF, Schadt EE (2012) This I believe: gaining new insights through integrating 'old' data. Front Genet 3: 137

44. Kumar V, Gu Y, Basu S, Berglund A, Eschrich SA, et al. (2012) Radiomics: the process and the challenges. Magn Reson Imaging 30(9): 1234-1248.

45. Mc Guire AL, Burke W (2008) An unwelcome side effect of direct-to-consumer personal genome testing: raiding the medical commons. JAMA 300(22): 2669-2671

46. Gamazon ER, Im HK, Duan S, Lussier YA, Cox NJ, et al. (2010) Exprtarget: an integrative approach to predicting human microRNA targets. PLoS One 15(10): e13534.

47. Denny JC, Ritchie MD, Basford MA, Pulley JM, Bastarache L, et al. (2010) PheWAS: demonstrating the feasibility of a phenome-wide scan to discover gene-disease associations. Bioinformatics 26(9): 1205-1210.

48. Nielsen FC, Borregaard N (2009) Pharmacogenetics and tailored drug therapy. Ugeskr Laeger, 171(10): 790-794.

49. Roederer MW, McLeod HL (2010) Applying the genome to national drug formulary policy in the developing world. Pharmacogenomics 11(5): 633-636.

50. Ong SE, Schenone M, Margolin AA, Li X, Do K, et al. (2009) Identifying the proteins to which small-molecule probes and drugs bind in cells. Proc Natl Acad Sci U S A 106(12): 4617-4622.

51. Petrosino JF (2018) The microbiome in precision medicine: the way forward. Genome Med 10(1): 12

52. Knox RJ, Friedlos F, Boland MP (1993) The bioactivation of CB 1954 and its use as a prodrug in antibody-directed enzyme prodrug therapy (ADEPT). Cancer Metastasis Rev 12(2): 195-212.

53. Li L (2017) Precision medicine in pharmacometrics and systems pharmacology. CPT pharmacometrics Syst Pharmacol 6(3): 151-152.

54. (2006) Centers for Disease Control and Prevention (CDC), Principles of Epidemiology in Public Health Practice: An introduction to Applied Epidemiology and Biostatistics. 
55. Nishi A, Milner DA, Giovannucci EL, Nishihara R, Tan AS, et al. (2016) Integration of molecular pathology, epidemiology and social science for global precision medicine. Expert Rev Mol Diagn 16(1): 11-23.
56. Ogino S, King EE, Beck AH, Sherman ME, Milner DA, et al. (2012) Interdisciplinary education to integrate pathology and epidemiology: towards molecular and population-level health science. Am J Epidemiol 176(8): 659-667. (c) (i) Creative Commons Attribution 4.0

For possible submissions Click Here NACS $\begin{gathered}\text { Novel } \\ \text { Approaches } \\ \text { in Cancer study }\end{gathered}$

Submit Article 